\title{
Antiviral and immunomodulatory interferon-beta in high-risk COVID-19 patients: a structured summary of a study protocol for a randomised controlled trial
}

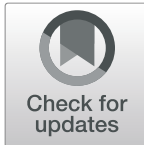

Eleonora Aricó ${ }^{1 * \dagger}$ (D), Luciano Castiello ${ }^{1+}$, Laura Bracci ${ }^{2}$, Francesca Urbani ${ }^{2,3}$, Flavia Lombardo ${ }^{4}$, Ilaria Bacigalupo ${ }^{4}$, Antonio Ancidoni ${ }^{4}$, Nicola Vanacore ${ }^{4}$, Alessandro Falcione ${ }^{5}$, Chiara Reggiani ${ }^{5}$, Giovanni Marco Dutti ${ }^{5}$, Maria Grazia Maglie ${ }^{5}$, Ombretta Papa ${ }^{5}$, Pier Luigi Bartoletti ${ }^{5}$, Giuseppina Ozzella ${ }^{6}$, Nazario Bevilacqua ${ }^{7}$, Emanuele Nicastri ${ }^{7 \dagger}$, Filippo Belardelli ${ }^{6 *}$ and Giuseppe Sconocchia ${ }^{6+}$

\begin{abstract}
Objectives: The primary objective of the study is to demonstrate the efficacy of low-dose IFN- $\beta$ in reducing the risk of SARS-CoV-2 recently infected elderly patients to progress towards severe COVID-19 versus control group within 28 days. Secondary objectives are:

1) To assess the reduction in Intensive Care Unit (ICU) admission in patients treated with IFN- $\beta$ versus control group within 28 days of randomization

2) To assess the reduction in number of deaths in IFN- $\beta$ compared to control group (day 28)

3) To evaluate the increase in proportion of participants returning to negative SARS-CoV-2 RT-PCR in IFN- $\beta$-treated versus control group at Day 14 and Day 28

4) To assess the increase in SARS-CoV-2-specific binding antibody titers in IFN- $\beta$ compared to control group (day 28)

5) To assess the safety of IFN- $\beta$-treated patients versus control group

Trial design: Randomized, Open-Label, Controlled, Superiority Phase II Study. Patients, who satisfy all inclusion criteria and no exclusion criteria, will be randomly assigned to one of the two treatment groups in a ratio 2:1 (IFNtreated versus control patients). Randomization will be stratified by gender. Stratified randomization will balance the presence of male and female in both study arms.
\end{abstract}

\footnotetext{
* Correspondence: eleonora.arico@iss.it; filippo.belardelli@ift.cnr.it

†Eleonora Aricò, Luciano Castiello, Emanuele Nicastri, Filippo Belardelli and Giuseppe Sconocchia contributed equally to this work.

${ }^{1}$ FaBioCell, Core Facilities, Istituto Superiore di Sanità, viale Regina Elena 299, 00161 Rome, Italy

${ }^{6}$ Institute of Translational Pharmacology, National Research Council, Via Fosso del Cavaliere 100, 00133 Rome, Italy

Full list of author information is available at the end of the article
}

(c) The Author(s). 2021 Open Access This article is licensed under a Creative Commons Attribution 4.0 International License, which permits use, sharing, adaptation, distribution and reproduction in any medium or format, as long as you give appropriate credit to the original author(s) and the source, provide a link to the Creative Commons licence, and indicate if changes were made. The images or other third party material in this article are included in the article's Creative Commons licence, unless indicated otherwise in a credit line to the material. If material is not included in the article's Creative Commons licence and your intended use is not permitted by statutory regulation or exceeds the permitted use, you will need to obtain permission directly from the copyright holder. To view a copy of this licence, visit http://creativecommons.org/licenses/by/4.0/ The Creative Commons Public Domain Dedication waiver (http://creativecommons.org/publicdomain/zero/1.0/) applies to the data made available in this article, unless otherwise stated in a credit line to the data. 
Participants: Male and female adults aged 65 years or older with newly diagnosed SARS-CoV-2 infection and mild COVID-19 symptoms are eligible for the study. The trial is being conducted in Rome.

Participants will be either hospitalized or home isolated. A group of physicians belonging to the Special Unit for Regional Continued Care (USCAR), specifically trained for the study and under the supervision of the National Institute for Infectious Diseases "Lazzaro Spallanzani", will be responsible for the screening, enrolment, treatment and clinical monitoring of patients, thus acting as a bridge between clinical centers and territorial health management.

Inclusion criteria are as follows:

- $\geq 65$ years of age at time of enrolment;

- Laboratory-confirmed SARS-CoV-2 infection as determined by PCR, in any specimen $<72$ hours prior to randomization;

- Subject (or legally authorized representative) provides written informed consent prior to initiation of any study procedures;

- Understands and agrees to comply with planned study procedures;

- Agrees to the collection of nasopharyngeal swabs and venous blood samples per protocol;

- Being symptomatic for less than 7 days before starting therapy;

- NEWS2 score $\leq 2$.

Exclusion criteria are as follows:

- Hospitalized patients with illness of any duration, and at least one of the following:

- Clinical assessment (evidence of rales/crackles on exam) and SpO2 $\leq 94 \%$ on room air at rest or after walking test,

OR

- Acute respiratory failure requiring mechanical ventilation and/or supplemental oxygen;

- Patients currently using IFN- $\beta$ (e.g., multiple sclerosis patients);

- Patients undergoing chemotherapy or other immunosuppressive treatments;

- Patients with chronic kidney diseases;

- Known allergy or hypersensitivity to IFN (including asthma);

- Any autoimmune disease (resulting from patient anamnesis);

- Patients with signs of dementia or neurocognitive disorders;

- Patients with current severe depression and/or suicidal ideations;

- Being concurrently involved in another clinical trial;

- HIV infection (based on the anamnesis);

- Use of any antiretroviral medication;

- Impaired renal function (eGFR calculated by CKD-EPI Creatinine equation $<30 \mathrm{ml} / \mathrm{min}$ );

- Presence of other severe diseases impairing life expectancy (e.g. patients are not expected to survive 28 days given their pre-existing medical condition);

- Any physical or psychological impediment in a patient that could let the investigator to suspect his/her poor compliance;

- Lack or withdrawal of informed consent

Intervention and comparator: Control arm: No specific antiviral treatment besides standard of care.

Treatment arm: $11 \mu \mathrm{g}$ (3MIU) of IFN- $\beta 1$ a will be injected subcutaneously at day 1, 3, 7, and 10 in addition to standard of care. The drug solution, contained in a pre-filled cartridge, will be injected by means of the RebiSmart ${ }^{\oplus}$ electronic injection device.

Interferon $\beta 1$ a (Rebif ${ }^{\oplus}$, Merck KGaA, Darmstadt, Germany) is a disease-modifying drug used to treat relapsing forms of multiple sclerosis (MS). The dose selected for this study is expected to exploit the antiviral and immunomodulatory properties of the cytokine without causing relevant toxicity or inducing refractoriness 
phenomena sometimes observed after high-dose and/or chronic IFN $\beta$ treatments.

Main outcomes: Primary endpoint of the study is the proportion of patients experiencing a disease progression, during at least 5 days, according to the National Early Warning Score (NEWS2). The NEWS2 score is a standardized approach aimed at promptly detecting signs of clinical deterioration in acutely ill patients and establishing the potential need for higher level of care. It is based on the evaluation of vital signs, including respiratory rate, oxygen saturation, temperature, blood pressure, pulse/heart rate, AVPU response. The resulting observations, compared to a normal range, are combined in a single composite "alarm" score. Any other clinical sign clearly indicating a disease worsening will be considered as disease progression.

Randomization: Sixty patients will be randomized 2:1 to receive IFN- $\beta 1$ a plus the standard of care or the standard of care only. Eligible patients will be randomized (no later than $36 \mathrm{~h}$ after enrolment) by means of a computerized central randomization system. All patients will receive a unique patient identification number at enrolling visit when signing the informed consent and before any study procedure is performed. This number remains constant throughout the entire study. The randomization of patients will be closed when 60 patients have been enrolled. The randomization will be stratified by sex; for each stratum a sequence of treatments randomly permuted in blocks of variable length (3 or 6 ) will be generated.

Blinding (masking): This is an open-label study. After the randomization, patients will be notified whether they will be in the experimental arm or in the control arm.

Numbers to be randomised (sample size): The study plans to enrol 60 patients: 40 in the IFN- $\beta 1$ a arm, 20 in the control arm, according to a 2:1 - treated: untreated ratio.

Trial Status: Protocol Version: 3.0

Version Date: 18/03/2021

The study is open for recruitment since 16/04/2021. Recruitment is expected to I be completed before 15/08/2021.

Trial registration: EudraCT No: 2020-003872-42, registration date: 19/10/2020.

Full protocol: The full protocol is attached as an additional file, accessible from the Trials website (Additional file 1). In the interest in expediting dissemination of this material, the familiar formatting has been eliminated; this Letter serves as a summary of the key elements of the full protocol."

Keywords: COVID-19, Randomised controlled trial, protocol, Interferon-beta, antiviral, immunomodulation, nonhospitalized patients

\section{Supplementary Information}

The online version contains supplementary material available at https://doi. org/10.1186/s13063-021-05367-6.

Additional file 1. Full study protocol.

\section{Acknowledgements}

We are grateful to Matilde Paggiolu and Pamela Papa for administrative support.

\section{Authors' contributions}

E.A., L.C., L.B., F.U., F.B. conceived and designed the trial; E.A., L.C., L.B., F.U., G.O., G.S. wrote the trial protocol and prepared the manuscript for submission; F.L., I.B., A.A., N.V., led the statistical analysis plan; O.P., P.L.B., N.B., E.N. provided clinical advice and revised the trial protocol. F.B. received funding by Merck KGaA. All authors approved the final version of the protocol and the manuscript.

\section{Funding}

The study is partially funded by Merck KGaA, who also provided the investigational drug. Merck has no role in study design, data collection, management, analysis, data interpretation, manuscript writing, or in the decision to submit manuscripts for publication.

Availability of data and materials Not applicable

\section{Declarations}

\section{Ethics approval and consent to participate}

The study was approved on 06/11/2020 by the Ethics Committee of the National Institute for Infectious Diseases "Lazzaro Spallanzani" in Rome, designed as National Committee for evaluation of clinical trials on human drugs in COVID-19 patients. Version 3, including non-substantial amendment to the original protocol, was approved on 23/03/2021 by the same committee.

Prior to inclusion, potential patients will first receive written and verbal information about the nature, purpose, procedures required by the protocol, possible risks and benefits of the study. Patients will have the time to carefully review the consent form and ask questions prior to completing. Informed consent to participate in the study will be obtained from all participants before enrolment. The original signed informed consent form will be retained in the patient's records and a copy of the informed consent form will be provided to the patient.

We herein certify that this trial has received ethical approval from the appropriate ethical committee as described above.

\section{Consent for publication}

Not applicable

\section{Competing interests}

The authors declare that they have no competing interests. 


\section{Author details}

'FaBioCell, Core Facilities, Istituto Superiore di Sanità, viale Regina Elena 299, 00161 Rome, Italy. ${ }^{2}$ Department of Oncology and Molecular Medicine, Istituto Superiore di Sanità, viale Regina Elena 299, 00161 Rome, Italy.

${ }^{3}$ Medical Biotechnology and Translational Medicine PhD School, II University of Rome "Tor Vergata", Via Montpellier 1, 00133 Rome, Italy. ${ }^{4}$ National Centre for Disease Prevention and Health Promotion, Istituto Superiore di Sanità, viale Regina Elena 299, 00161 Rome, Italy. ${ }^{5}$ Special Units for Regional Continued Care (USCAR), Rome, Italy. Institute of Translational Pharmacology, National Research Council, Via Fosso del Cavaliere 100, 00133 Rome, Italy. "National Institute for Infectious Diseases "Lazzaro Spallanzani", Via Portuense 292, 00149 Rome, Italy.

Received: 3 June 2021 Accepted: 9 June 2021

Published online: 03 September 2021

\section{Publisher's Note}

Springer Nature remains neutral with regard to jurisdictional claims in published maps and institutional affiliations.

Ready to submit your research? Choose BMC and benefit from:

- fast, convenient online submission

- thorough peer review by experienced researchers in your field

- rapid publication on acceptance

- support for research data, including large and complex data types

- gold Open Access which fosters wider collaboration and increased citations

- maximum visibility for your research: over $100 \mathrm{M}$ website views per year

At BMC, research is always in progress.

Learn more biomedcentral.com/submissions 